\title{
Inhibition of the growth of hepatocellular carcinoma cells through fibroblast growth factor 18 suppressed by miR-139
}

\author{
LI YANG ${ }^{*}$, DIAN YIN* ${ }^{*}$ YILANG WANG and LILI CAO \\ Department of Oncology, The First People's Hospital of Nantong, Nantong, Jiangsu 226001, P.R. China
}

Received February 15, 2017; Accepted July 19, 2017

DOI: $10.3892 /$ or.2017.5869

\begin{abstract}
Hepatocellular carcinoma (HCC) is a highly malignant tumor and one of the most common causes for human cancer-related deaths. Fibroblast growth factor 18 (FGF18) is overexpressed in many types of cancer, and is associated with cell proliferation, invasion and angiogenesis. miR-139 has recently been reported as a tumor suppressor in various types of cancer and it can regulate many tumor-related genes, however its association with FGF18 expression in HCC has not been reported and thus remains unknown. In the present study, to explore the potential regulation mechanism of miR-139 with FGF18 in HCC, HCC tissues and cell lines were used. The results revealed that FGF18 was highly expressed in HCC tissues and cells, however miR-139 was lowly expressed. FGF18 was demonstrated to be a direct target of miR-139. Furthermore, the suppressive effect of miR-139 on FGF18 and in turn on proliferation, apoptosis, invasion, migration and tumor-induced angiogenesis of HCC cells was investigated. FGF18 was suggested as a prognostic biomarker and therapeutic target in HCC patients and miR-139 may be a promising strategy used in HCC treatment via the suppression of FGF18.
\end{abstract}

\section{Introduction}

Hepatocellular carcinoma (HCC) is one of the six malignancies in the world and with a mortality rate among patients $(1,2)$. Although advances in medicine and surgical procedures have improved the treatment of HCC, the prognosis of patients with HCC is poor with a low long-term survival rate due to a high recurrence rate and postoperative metastasis (3-5). The accurate molecular mechanism that contributes to the occurrence and development of HCC is unclear. Thus, it is necessary to

Correspondence to: Dr Li Yang, Department of Oncology, The First People's Hospital of Nantong, 6 North Hai'er Xiang Road, Nantong, Jiangsu 226001, P.R. China

E-mail: ntyyzlkyl@163.com

*Contributed equally

Key words: hepatocellular carcinoma, fibroblast growth factor 18, miR-139, growth find new individualized molecules which are involved in HCC and clarify their roles, to further develop new therapeutics for HCC treatment.

FGF18 is a member of the fibroblast growth factor (FGF) family (6). The FGF family includes 22 members, which possess mitogenic and cell survival activities, and are involved in a variety of biological processes, such as embryonic development, cell growth, morphogenesis, tissue repair and angiogenesis (7). FGF18 is an essential mitogen in embryonic limb development and is required for differentiation during osteogenesis and chondrogenesis (8-10). Additionally, as a pleiotropic growth factor, FGF18 stimulates proliferation in a number of tissues, most notably the liver (11) and small intestine $(8,12)$. Recently, FGF18 was reported to be highly expressed in colon cancer and ovarian cancer. Increased FGF18 expression, which plays a key role in controlling tumor growth and invasion, was associated with tumor progression and poor overall survival in patients (13-15). FGF18 can also affect both the tumor and the connective tissue cells of the tumor microenvironment $(16,17)$. Overexpression of FGF18 promoted tumor progression by modulating migration, invasion and tumorigenicity of cancer cells arguably through different signaling pathways $(13,15)$. These findings demonstrated that FGF18 is important for a subset of ovarian cancers and may serve as a therapeutic target. However, there is still no study concerning the regulation mechanism of FGF18 in HCC.

The occurrence of cancers undergoes multiple complicated stages that are caused by many factors. Thus, there is a lack of effective treatments. The strategy of gene-direct inhibition may be an effective method in cancer therapy. MicroRNAs (miRNAs) are a class of small RNAs that can regulate gene expression at the mRNA level and function in various cellular processes and biological activities. They can be used to regulate the development of cancer, through cell apoptosis, invasion and proliferation (18). Numerous miRNAs in HCC have been widely investigated, and have also been identified as diagnostic and prognostic markers as well as therapeutic candidates for HCC. This strategy may provide accurate clinical decisions for HCC diagnosis and treatment (19,20).

In the present study, we demonstrated that the expression of FGF18, which was investigated in $\mathrm{HCC}$, was regulated by miR-139. In addition, the effects of FGF18 suppression by miR-139 on the biological processes of HCC cells were also revealed. 


\section{Materials and methods}

Clinical specimens, cell culture and transfection. Human hepatocellular carcinoma (HCC) and matched adjacent non-tumor tissues were collected from 15 patients at the Department of Oncology, The First People's Hospital of Nantong. The present study was approved by the Institute Research Ethics Committee of the First People's Hospital of Nantong. Human HCC cell lines HepG2, Huh7 and normal control liver cell line LO2, as well as human embryonic kidney 293 (HEK293) cells were all obtained from Biomics Biotechnologies Co., Ltd. (Nantong, China), cultured in Dulbecco's modified Eagle's medium (DMEM) and supplemented with $10 \%$ fetal bovine serum (FBS) (Thermo Fisher Scientific, Inc., Waltham, MA, USA) at $37^{\circ} \mathrm{C}$ in a humidified incubator with $5 \% \mathrm{CO}_{2}$.

miR-139 mimics were used for upregulation of endogenous miR-139, and a miR-139 sequence-scrambled RNA was used as a negative control miRNA (NC_miR). Lipofectamine ${ }^{\circledR} 2000$ transfection reagent (Thermo Fisher Scientific, Inc.) was used for transfection of miRNAs into cells according to the manufacturer's instructions. miR-139 mimics and NC_miR were obtained from Biomics Biotechnologies Co., Ltd. (Nantong, China).

Dual-luciferase reporter assay. The 3' untranslated region (3'UTR) region complementary DNA sequence of human FGF18 mRNA (NCBI Reference Sequence: NM_003862.2) was amplified by polymerase chain reaction (PCR) and constructed into the pGL3-vector (Promega, Madison, WI, USA) as a dual-luciferase miRNA target expression vector (wild-type FGF18 3'UTR, wtFGF18-3'UTR) to evaluate miR-139 activity in cells. The FGF18 3'UTR mutant vector was also constructed as the negative control (mutant FGF18 3'UTR, mFGF18-3'UTR). Briefly, HEK293 cells were seeded into a 24-well plate and grown for $24 \mathrm{~h}$. Then wtFGF18-3'UTR or mFGF18-3'UTR was co-tansfected with miR-139 mimics or NC_miR using Lipofectamine ${ }^{\circledR} 2000$ transfection reagent (Thermo Fisher Scientific, Inc.); pRL-TK (Promega) was co-transfected as an internal control. After transfection for $48 \mathrm{~h}$, cells were collected and lysed, firefly and Renilla luciferase activities in cell lysates were detected using the DLR assay system (Promega) according to the manufacturer's instructions.

Real-time quantitative PCR (RT-qPCR). After cells were treated with miRNAs as aforementioned, the total RNA of cells was extracted using TRIzol ${ }^{\circledR}$ reagent (Thermo Fisher Scientific, Inc.) and small RNA enriched with miRNAs was isolated using mirPremier ${ }^{\circledR}$ microRNA isolation kit (Sigma-Aldrich, St. Louis MO, USA) according to the manufacturer's instructions. Detection of miRNA expression levels in tissues or cells was performed by stem-loop RT-qPCR (21). U6 small RNA or GAPDH was used as an internal control for miRNA or mRNA detection, respectively. RT-qPCR reactions were carried out using the SYBR-Green One-Step RT-qPCR kit (Thermo Fisher Scientific, Inc.) according to the manufacturer's instructions. The relative miRNA or mRNA expression levels were evaluated by $2^{-\Delta \Delta C t}$ method (22). The primer sequences were FGF18 forward, 5'-ACCTTCGGTAGTCAAGTC-3' and reverse, 5'-CGTGTAGTTGTTCTCCAG-3'; GAPDH forward,
5'-GAGTCCACTGGCGTCTTC-3' and reverse, 5'-GATGAT CTTGAGGCTGTTGTC-3'.

Western blot analysis. Cells were plated in a 6-well plate, after treatment for $48 \mathrm{~h}$ as aforementioned. The cells were collected and lysed in a cell RIPA lysis and extraction buffer (Thermo Fisher Scientific, Inc.) on ice. Centrifugation to collect the proteins, then separation by polyacrylamide gel electrophoresis (PAGE) and transfer to a polyvinylidene difluoride (PVDF) membrane (Millipore, Billerica, MA, USA) followed. Subsequently, the membrane was incubated with a primary antibody rabbit anti-human FGF18 (1:1,000 dilution) or a mouse anti-human $\beta$-actin antibody (1:5,000 dilution) (all from Abcam, Cambridge, MA, USA) as an internal control. After being washed with Tris-buffered saline and Tween-200 (TBST) the membrane was incubated with a horseradish peroxidase-conjugated secondary antibody (1:5,000 dilution) (Abcam) for $1.5 \mathrm{~h}$ at room temperature, and then washed in TBST. The specific proteins were detected with ECL substrate (Thermo Fisher Scientific, Inc.).

Cell proliferation assay. The proliferation abilities of cells were assessed using Cell Counting Kit-8 (CCK-8) (Sigma-Aldrich). Briefly, $5 \times 10^{3}$ cells/well were seeded on a 96-well plate before treatment and grown to $\sim 70 \%$ confluence for $24 \mathrm{~h}$. Post-treatment at $0,24,48,72$ and $96 \mathrm{~h}$, the cells were washed with phosphate-buffered saline (PBS) three times, and replaced with $100 \mu \mathrm{l} /$ well of PBS, and then $10 \mu \mathrm{l}$ of FCCK working solution was added to each well. After incubation at $37^{\circ} \mathrm{C}$ in an incubator for $30 \mathrm{~min}$, the fluorescence intensity of each well was assessed at $535 \mathrm{~nm}$ using a fluorescence microplate reader (BioTek, Winooski, VT, USA).

Cell apoptosis assay. Cell apoptosis was determined by flow cytometric (FCM) analysis using Annexin V-FITC/propidium iodide (PI) double staining (Sigma-Aldrich). The cells were seeded on a 6-well plate for $24 \mathrm{~h}$ of growth and treated as aforementioned for $48 \mathrm{~h}$. Briefly, $1 \times 10^{5}$ cells/well were harvested and washed with PBS, and then re-suspended in Annexin-binding buffer, followed by incubation with FITC-conjugated Annexin $\mathrm{V}$ and propidium iodide (PI) for $15 \mathrm{~min}$ at room temperature. Finally, the cells were analyzed by FCM (BD Biosciences, Franklin Lakes, NJ, USA).

Cell migration assay. A cell wound scratch assay was used to determine the migration abilities of HCC cells. Briefly, $1 \times 10^{4}$ cells/well were plated on a 12 -well plate for $24 \mathrm{~h}$ of growth and treated as aforementioned. Cell wounds were made to confluent monolayer cells with a pipette tip post 48-h treatment and the cells were washed with DMEM twice. Cell migration was monitored at 0 and $48 \mathrm{~h}$ after the scratch.

Cell invasion assay. The invasion abilities of cells were detected by Transwell assay. Cells/well $\left(2 \times 10^{5}\right)$ were seeded into a 24-well plate for $24 \mathrm{~h}$ and treated for $48 \mathrm{~h}$, then suspended in DMEM at a density of $1 \times 10^{6}$ cells $/ \mathrm{ml}$. Transwell chambers (Corning, NY, USA) were incubated with DMEM for $1 \mathrm{~h}$ before treatments; the upper and lower chamber was separated by an $8-\mu \mathrm{m}$ pore polycarbonate membrane which was coated with $50 \mu 1$ of $0.5 \mathrm{mg} / \mathrm{ml}$ Matrigel (BD Biosciences). A cell 
A

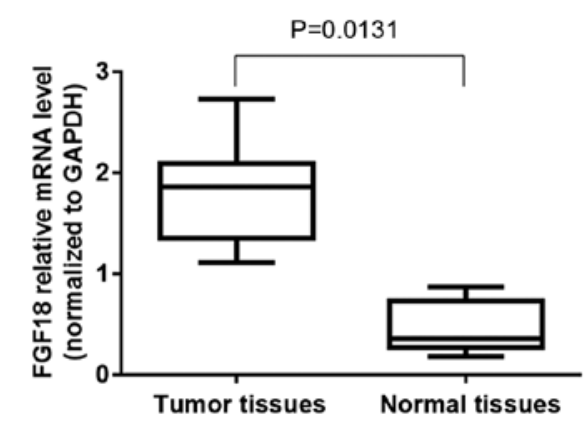

C

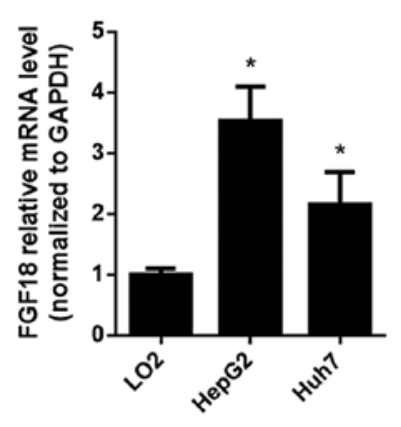

B
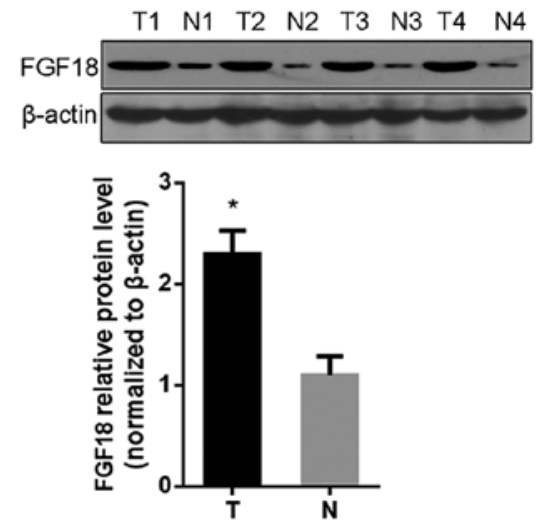

D
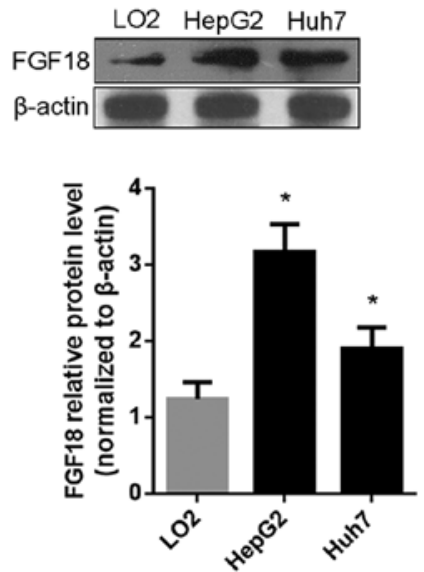

Figure 1. High expression of FGF18 in HCC tissues and cell lines. (A) FGF18 mRNA levels in 15 cases of HCC tissues and matched adjacent normal tissues were detected by RT-qPCR. (B) FGF18 protein levels in 15 cases of HCC tissues and matched adjacent normal tissues was detected by western blotting. (C) The mRNA expression level of FGF18 in HCC cell lines (HepG2 and Huh7) was detected by RT-qPCR, and compared with the expression level in LO2 cells; ${ }^{*} \mathrm{P}<0.05$. (D) The protein level of FGF18 in HCC cells (HepG2 and Huh7) was detected by western blotting and compared with the expression level in LO2 cells; ${ }^{*} \mathrm{P}<0.05$. HCC, hepatocellular carcinoma; FGF18, fibroblast growth factor 18.

suspension of $100 \mu \mathrm{l}$ was added into each upper chamber with $600 \mu 1$ DMEM containing 10\% FBS or conditioned medium which was the cell supernatant with treatment as aforementioned for $48 \mathrm{~h}$. The cells on the top surface of the membrane were carefully removed using cotton swabs post-treatment for $24 \mathrm{~h}$. The cells on the Transwell chambers were fixed in $10 \%$ formaldehyde for $30 \mathrm{sec}$, and then stained with $0.5 \%$ crystal violet solution. After being washed with PBS, the cells on the top surface of the membrane were carefully removed again, and the cells on the bottom surface of the membrane were observed and counted under a microscope.

Tube formation assay. An in vitro angiogenesis model was used to evaluate the inhibitory effects of miRNAs on angiogenesis. Briefly, a 24-well plate was coated with $100 \mu \mathrm{l} /$ well Matrigel (BD Biosciences) and incubated at $37^{\circ} \mathrm{C}$ for $30 \mathrm{~min}$. Human umbilical vein endothelial cells (HUVECs) at a concentration of $1 \times 10^{5}$ cells/well were re-suspended in conditioned medium (which was the supernatant of the cells treated with miRNAs for $48 \mathrm{~h}$ ), then seeded on a Matrigel coated 24-well plate and cultured in a $37^{\circ} \mathrm{C} / 5 \% \mathrm{CO}_{2}$ incubator for $24 \mathrm{~h}$. The number of branching points was counted under a microscope.

Statistical analysis. All the experiments were performed independently three times. The data are shown as the mean values \pm standard deviation (SD). Statistical analyses were performed using SPSS 19.0 software (SPSS, Inc., Chicago, IL, USA), and the results were analyzed using one way ANOVA followed by post hoc test to assess statistical significance. All P-values were based on a two-side statistical analysis and $\mathrm{P}<0.05$ was considered to indicate statistical significance.

\section{Results}

FGF18 expression in human HCC patients and cells. The expression of FGF18 was detected by RT-qPCR and western blot assay in 15 cases of HCC tissues and matched adjacent normal tissues, as well as in HCC cell lines HepG2 and Huh7. Compared with normal tissues, the results revealed that FGF18 mRNA and protein levels were highly expressed in HCC patients $(\mathrm{P}=0.0131)$ (Fig. $1 \mathrm{~A}$ and $\mathrm{B})$. Moreover, the mRNA and protein levels of FGF18 were also highly expressed in HCC cell lines HepG2 and Huh7, compared with normal liver cell LO2 ( $\mathrm{P}<0.05)$ (Fig. 1C and D).

Expression profile of miR-139 in human HCC patients and cells. The expression of miR-139 was detected by RT-qPCR both in HCC tissues and cell lines. The results revealed that, miR-139 was significantly decreased in HCC tissues $(\mathrm{P}=0.0091)$ (Fig. 2A), compared with normal tissues, it was 
A

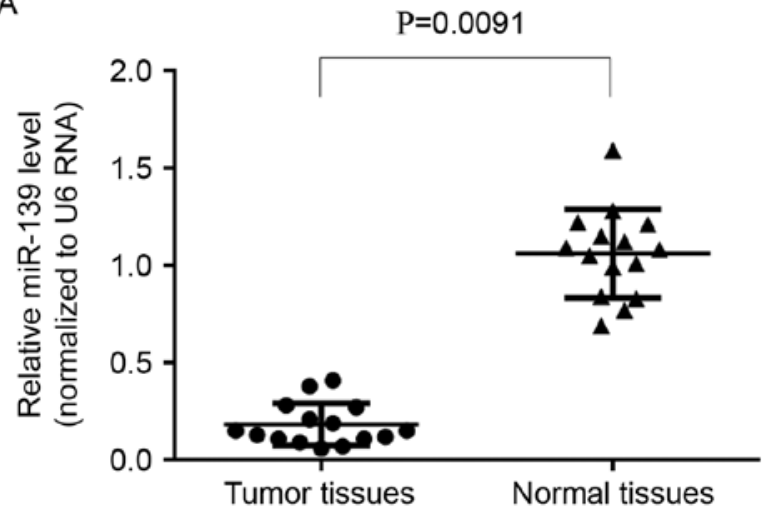

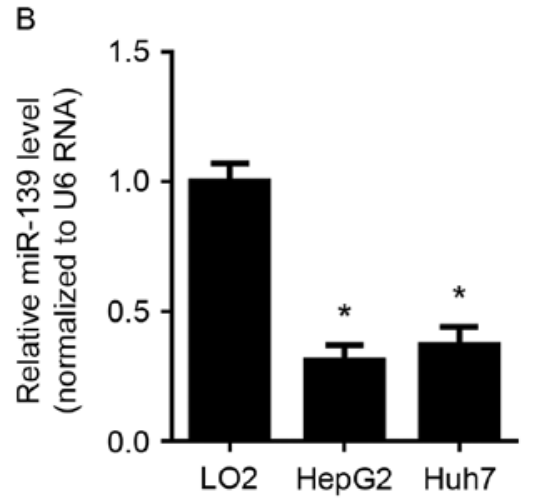

Figure 2. Low expression of miR-139 in HCC tissues and cell lines. (A) The expression of miR-139 in 15 cases of HCC tissues and matched adjacent normal tissues was detected by RT-qPCR. (B) The miR-139 expression level in HCC cell lines (HepG2 and Huh7) was detected by Stem-loop RT-qPCR and compared with the expression level in LO2 cells; ${ }^{*} \mathrm{P}<0.05$. HCC, hepatocellular carcinoma.

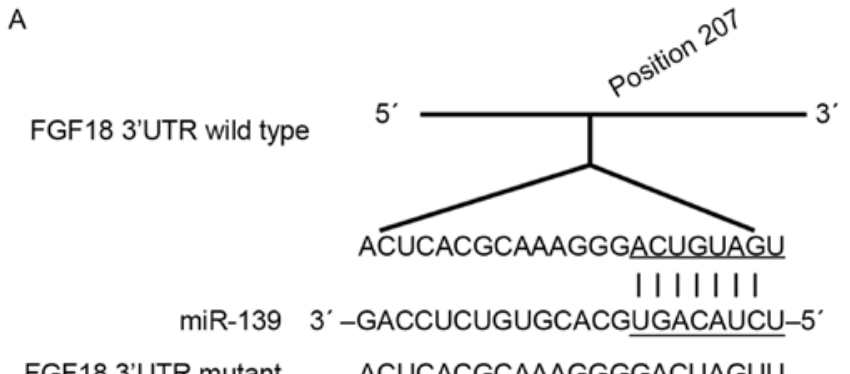

FGF18 3'UTR mutant ACUCACGCAAAGGGGACUAGUU

B

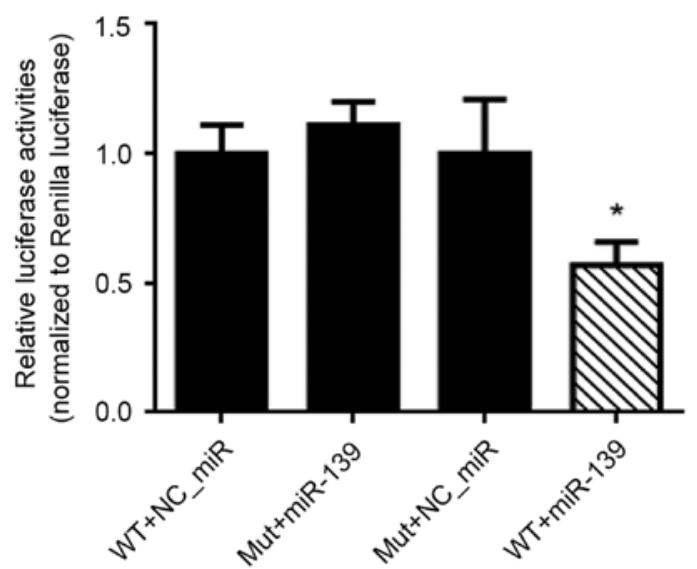

Figure 3. miR-139 targets the 3'UTR region of FGF18 mRNA. (A) Predicted base pairing of miR-139 seed sequences in the 3'UTR of FGF18 by online software. (B) Relative suppression of firefly luciferase expression of FGF18 luciferase reporter vector by miR-139; ${ }^{*} \mathrm{P}<0.05$. FGF18, fibroblast growth factor 18.

also lowly expressed in both HepG2 and Huh7 cells, compared with that in $\mathrm{LO} 2$ cells $(\mathrm{P}<0.05)$ (Fig. 2B).

miR-139 targeting the 3'UTR of FGF18 mRNA. The target site of miR-139 with the position 207 of the FGF18 mRNA sequence was predicated by web online software (http://www. targetscan.org), and then the dual-luciferase reporter (DLR) assay was used to observe the site binding and inhibition effects. The reporter vectors of the wild-type wtFGF18-3'UTR and mutant-type mFGF18-3'UTR were constructed (Fig. 3A). The result of the DLR assay revealed that co-tansfection of HEK293 cells with miR-139 mimics and wtFGF18-3'UTR, resulted in a marked decrease of luciferase activities, compared with NC_miR transfected with wtFGF18-3'UTR or mFGF18-3'UTR, and miR-139 transfected with mFGF183'UTR (Fig. 3B).

Inhibition of FGF18 by miR-139 in HCC cells. The inhibitory effect of miR-139 on the expression of FGF18 was detected by western blot assay. Compared with NC_miR-treated cells, the results revealed that the FGF18 protein levels were suppressed by miR-139 in both HCC cells HepG2 and Huh7 $(\mathrm{P}<0.05)$ (Fig. 4A and B).

The growth effect of FGF18 on HCC cells is inhibited by $m i R-139$. To investigate the inhibition effect of miR-139 on the growth of HCC cells, a CCK8 assay was performed. The results revealed that the growth of HCC cell lines, HepG2 and Huh7, were both inhibited by miR-139 at 48 and $72 \mathrm{~h}$, compared with the NC_miR-treated cells $(\mathrm{P}<0.05)$ (Fig. 5).

Apoptosis effect of FGF18 on HCC cells is inhibited by $m i R-139$. The inhibitory effect of miR-139 on the apoptosis of HCC cells was determined by flow cytometric (FCM) analysis after Annexin V-FITC/PI double staining. The results revealed that the apoptosis of HCC cell lines, HepG2 and Huh7, were both inhibited by miR-139 when compared with the NC_miR-treated cells $(\mathrm{P}<0.05)$ (Fig. 6).

Invasion and migration effect of FGF18 on HCC cells is inhibited by miR-139. To observe the inhibitory effect of invasion and migration abilities, Transwell and cell wound scratch assays were separately used. The result of the Transwell assay revealed that the invasion abilities of HCC cell lines, HepG2 and Huh7, were decreased significantly after miR-139 treatment, compared with the NC_miR-treated cells $(\mathrm{P}<0.05)$ (Fig. 7A). Similarly, the result of the cell wound scratch assay revealed that the migratory abilities of HCC cell lines, HepG2 and Huh7, were also markedly decreased postmiR-139 treatment, compared with the NC_miR-treated cells $(\mathrm{P}<0.05)$ (Fig. 7B). 
A
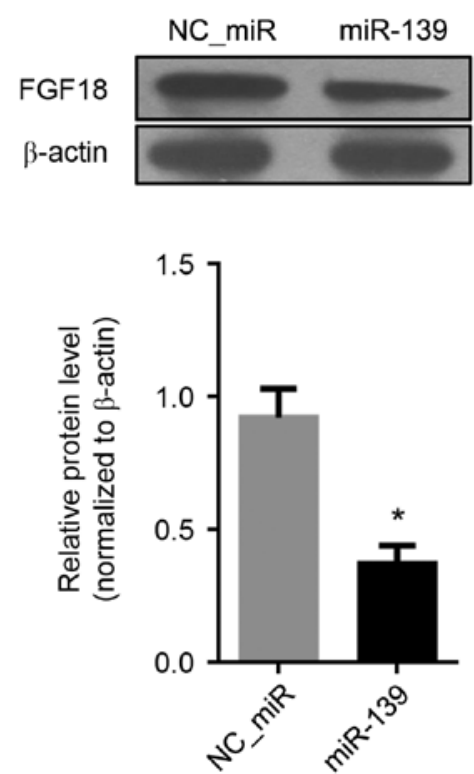
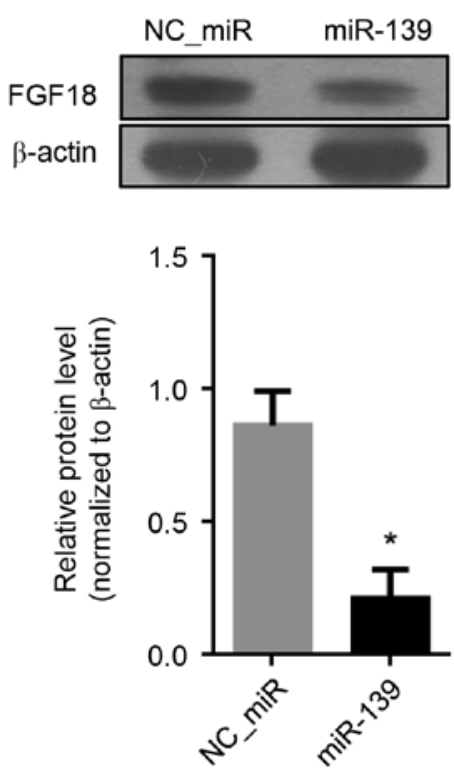

Figure 4. Inhibitory effects of miR-139 on the expression of FGF18 was detected by western blot analysis. (A) The protein level of FGF18 was inhibited by miR-139 in HepG2 cells. (B) The protein level of FGF18 was inhibited by miR-139 in Huh7 cells. FGF18, fibroblast growth factor 18.
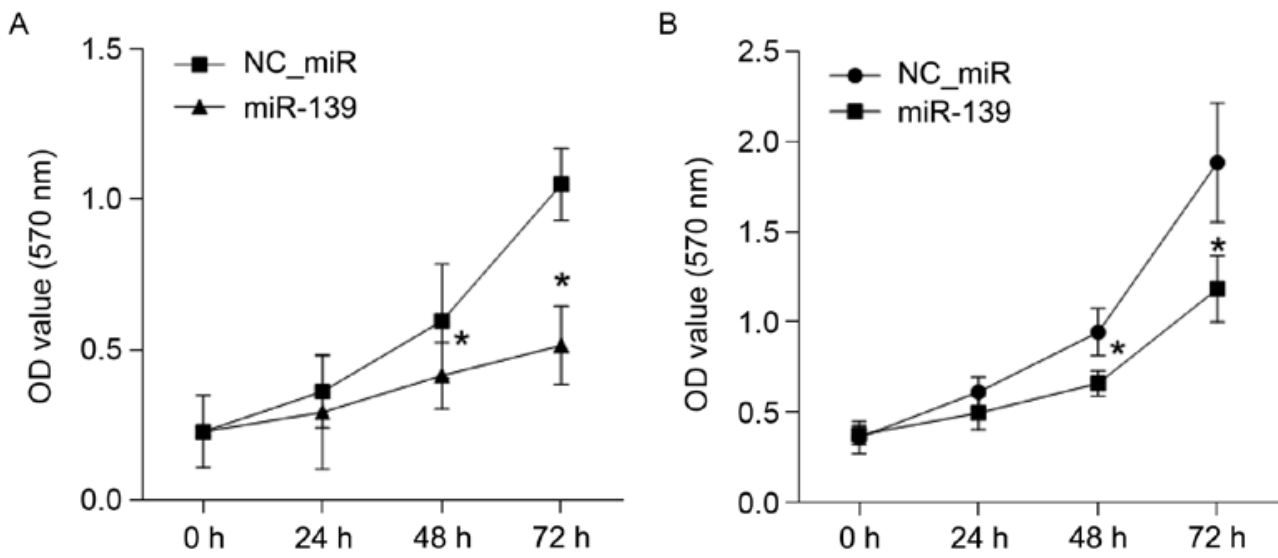

Figure 5. Inhibitory effects of miR-139 on the proliferation of HCC cells were detected by CCK8 assay. (A) The proliferation ability of HepG2 cells was inhibited by miR-139. (B) The proliferation of Huh7 cells was inhibited by miR-139; "P<0.05, miR-139-treated cells compared with the NC_miR-treated cells. HCC, hepatocellular carcinoma.

A

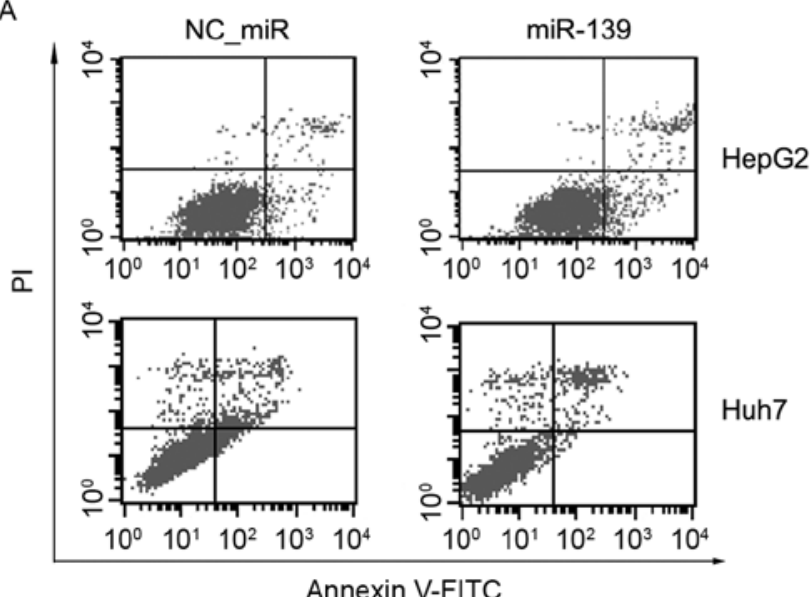

B

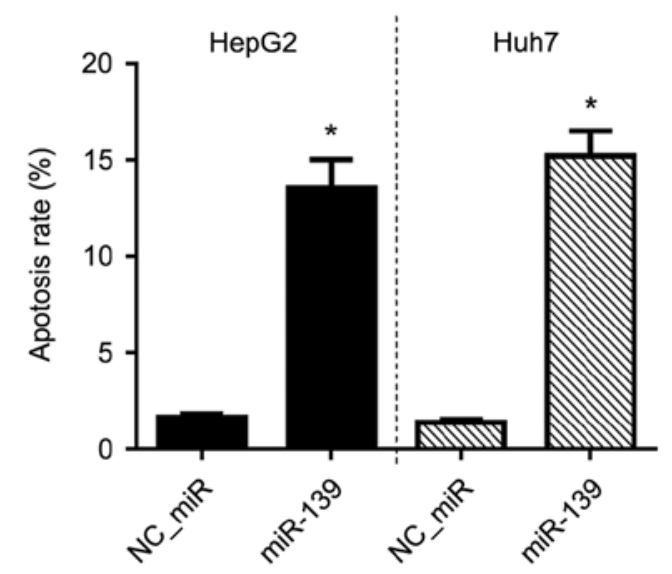

Figure 6. Inhibitory effects of miR-139 on the apoptosis of HCC cells. (A) Cell apoptosis was assessed by flow cytometric analysis after Annexin V-FITC/PI staining. (B) The cell apoptosis rate presented by Annexin V-FITC and PI double stained-positive cells; ${ }^{*} \mathrm{P}<0.05$, miR-139-treated cells compared with the NC_miR-treated cells. HCC, hepatocellular carcinoma. 
A
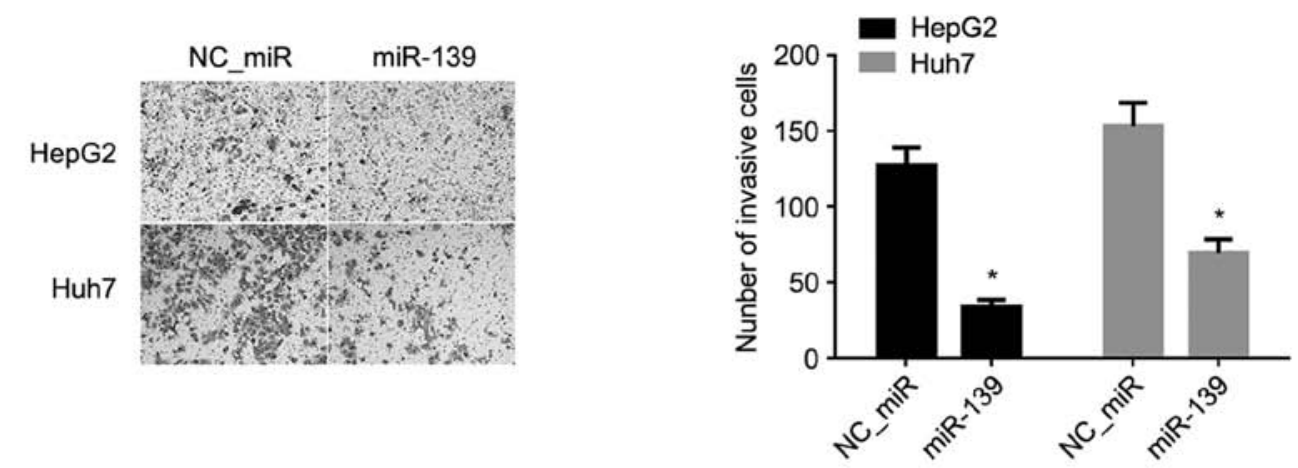

B
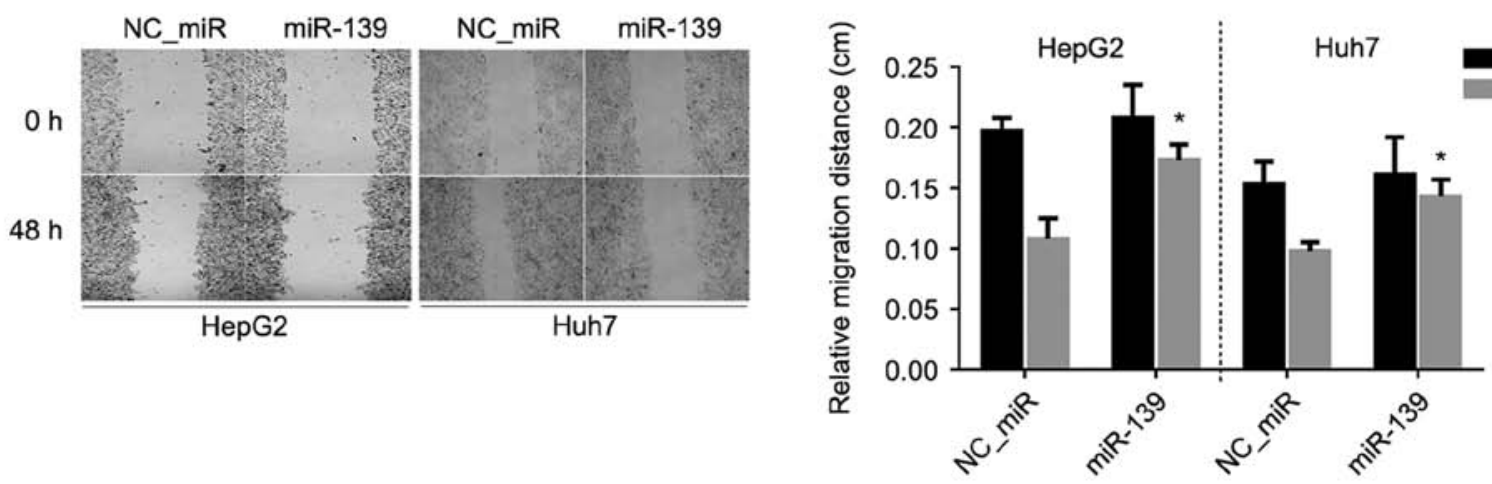

C
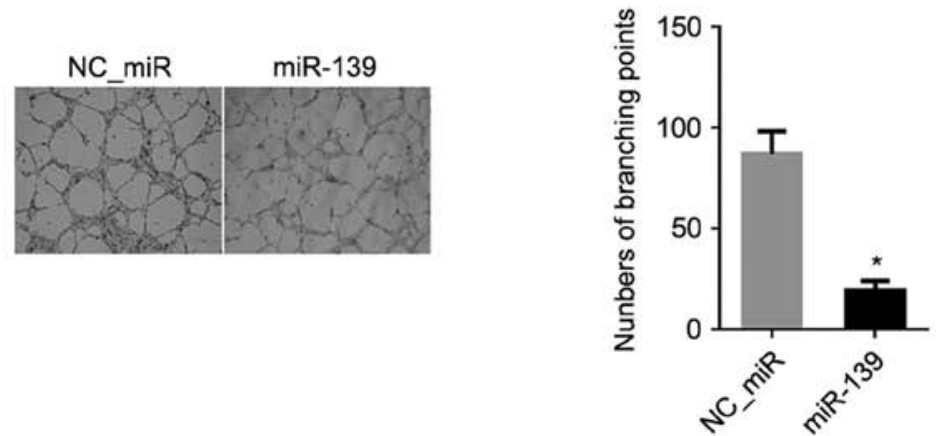

Figure 7. Inhibitory effects of miR-139 on the abilities of invasion, migration and tube formation in HCC cells. (A) The invasion abilities of HepG2 and Huh7 cells were inhibited by miR-139 as detected by Transwell assay. (B) The migration abilities of HepG2 and Huh7 cells were inhibited by miR-139 as detected by cell wound scratch assay. (C) Tube formation was induced by HepG2 or Huh7 cells inhibited by miR-139 in a HUVEC angiogenesis model; * $<0.05$, miR-139treated cells compared with the NC_miR-treated cells. HCC, hepatocellular carcinoma; HUVEC, human umbilical vein endothelial cell.

Tube formation effect of FGF18 on HCC cells is inhibited by miR-139. Tube formation was evaluated in a HUVEC angiogenesis model. HUVECs stimulated by the conditioned medium derived from $\mathrm{HCC}$ cells were transfected with miR-139 mimics and NC_miR. HUVECs treated with the conditioned medium of miR-139 mimics were significantly inhibited to form extensive and enclosed tube networks and when compared with the untreated cells, the number of branching points was less than the NC_miR-treated cells $(\mathrm{P}<0.05)$ (Fig. 7C).

\section{Discussion}

High expression of fibroblast growth factor 18 (FGF18) is related to the development and prognosis in several types of cancer, and has a relationship with the cell proliferation, invasion and angiogenesis (13-15). However, its expression and functions in HCC was still unclear. In the present study, high expression of FGF18 on the mRNA and protein level was demonstrated in HCC tissues and cell lines, such as HepG2 and Huh7 (Fig. 1).

miRNAs are a class of small RNAs 19-23 nt in length (23), that have been reported as novel biomarkers in many carcinomas and that play essential roles in carcinogenesis (24). Evidence has suggested that miRNAs can act as tumor suppressors or promoters in various carcinomas (25). Recent studies indicated that miR-139 was reported as a tumor suppressor in many types of cancer, such as breast cancer (26), acute myeloid leukemia (27), bladder (28), colorectal (29), cervical (30) and lung cancer (31). miR-139 was lowly expressed in these cancers, while highly expressed in normal tissues or cells. miR-139 was found to induce cell apoptosis, suppress the tumor growth and decrease tumor metastasis and drug sensitivity by inhibiting the EMT signaling pathway (30,32). Overexpression of miR-139-5p resulted in the inhibition of uterine leiomyoma cell growth (34). It suppressed glioma cell proliferation and regulated the cell 
cycle (34). In the present study, we also found that miR-139 was lowly expressed in HCC tumor tissues (Fig. 2A) and cell lines (both in HepG2 and Huh7) (Fig. 2B).

Furthermore, the relationship between FGF18 and miR-139 was investigated and predication of software revealed that miR-139 can bind to the mRNA of FGF18 (Fig. 3A). The region of the binding site was synthesized and cloned into a dual-luciferase reporter vector and was tansfected with miR-139 mimics into HEK293 cells. The results revealed that FGF18 was directly inhibited by miR-139 (Fig. 3B), suggesting that FGF18 was the target of miR-139.

Additionally, to explore whether FGF18 can be regulated by miR-139 in HCC cells, HCC cell lines, HepG2 and Huh7 were used. In both cell lines, a high expression of miR-139 (transfection of miR-139 mimics) resulting in a decrease of the protein level of FGF18 (Fig. 4), indicated that FGF18 can be suppressed by miR-139 in HCC cells. The biological functions of miR-139 influencing HCC cells were also demonstrated. After HepG2 and Huh7 cells were treated with miR-139, cell proliferation (Fig. 5), apoptosis (Fig. 6), invasion and migration were all inhibited, and the ability of angiogenesis induction was also decreased (Fig. 7).

In brief, the results of the present study reveal that FGF18, and miR-139 may be biomarkers or novel prognostic indicators in HCC, and inhibition of FGF18, particularly by miR-139 may be a potential approach for HCC treatment.

\section{References}

1. Torre LA, Bray F, Siegel RL, Ferlay J, Lortet-Tieulent J and Jemal A: Global cancer statistics, 2012. CA Cancer J Clin 65: 87-108, 2015.

2. Ferlay J, Soerjomataram I, Dikshit R, Eser S, Mathers C, Rebelo M, Parkin DM, Forman D and Bray F: Cancer incidence and mortality worldwide: Sources, methods and major patterns in GLOBOCAN 2012. Int J Cancer 136: E359-E386, 2015.

3. Wang C, Zhang F, Fan H, Peng L, Zhang R, Liu S and Guo Z: Sequence polymorphisms of mitochondrial D-loop and hepatocellular carcinoma outcome. Biochem Biophys Res Commun 406: 493-496, 2011.

4. Forner A, Llovet JM and Bruix J: Hepatocellular carcinoma. Lancet 379: 1245-1255, 2012

5. Shindoh J, Hasegawa K, Inoue Y, Ishizawa T, Nagata R, Aoki T, Sakamoto Y, Sugawara Y, Makuuchi M and Kokudo N: Risk factors of post-operative recurrence and adequate surgical approach to improve long-term outcomes of hepatocellular carcinoma. HPB Oxf 15: 31-39, 2013.

6. Hu MC, Qiu WR, Wang YP, Hill D, Ring BD, Scully S, Bolon B, DeRose M, Luethy R, Simonet WS, et al: FGF-18, a novel member of the fibroblast growth factor family, stimulates hepatic and intestinal proliferation. Mol Cell Biol 18: 6063-6074, 1998.

7. Cinque L, Forrester A, Bartolomeo R, Svelto M, Venditti R, Montefusco S, Polishchuk E, Nusco E, Rossi A, Medina DL, et al: FGF signalling regulates bone growth through autophagy. Nature 528: 272-275, 2015.

8. Liu Z, Lavine KJ, Hung IH and Ornitz DM: FGF18 is required for early chondrocyte proliferation, hypertrophy and vascular invasion of the growth plate. Dev Biol 302: 80-91, 2007.

9. Hung IH, Schoenwolf GC, Lewandoski M and Ornitz DM: A combined series of Fgf9 and Fgfl8 mutant alleles identifies unique and redundant roles in skeletal development. Dev Biol 411: 72-84, 2016.

10. Dahlberg LE, Aydemir A, Muurahainen N, Gühring H, Fredberg Edebo H, Krarup-Jensen N, Ladel CH and Jurvelin JS: A first-in-human, double-blind, randomised, placebo-controlled, dose ascending study of intra-articular rhFGF18 (sprifermin) in patients with advanced knee osteoarthritis. Clin Exp Rheumatol 34: 445-450, 2016.

11. Itoh N, Nakayama Y and Konishi M: Roles of FGFs as paracrine or endocrine signals in liver development, health, and disease. Front Cell Dev Biol 4: 30, 2016.
12. Davidson D, Blanc A, Filion D, Wang H, Plut P, Pfeffer G, Buschmann MD and Henderson JE: Fibroblast growth factor (FGF) 18 signals through FGF receptor 3 to promote chondrogenesis. J Biol Chem 280: 20509-20515, 2005.

13. Koneczny I, Schulenburg A, Hudec X, Knöfler M, Holzmann K, Piazza G, Reynolds R, Valent P and Marian B: Autocrine fibroblast growth factor 18 signaling mediates Wnt-dependent stimulation of CD44-positive human colorectal adenoma cells. Mol Carcinog 54: 789-799, 2015.

14. El-Gendi S, Abdelzaher E, Mostafa MF and Sheasha GA: FGF18 as a potential biomarker in serous and mucinous ovarian tumors. Tumour Biol 37: 3173-3183, 2016.

15. Wei W, Mok SC, Oliva E, Kim SH, Mohapatra G and Birrer MJ: FGF18 as a prognostic and therapeutic biomarker in ovarian cancer. J Clin Invest 123: 4435-4448, 2013.

16. Sonvilla G, Allerstorfer S, Stättner S, Karner J, Klimpfinger M, Fischer H, Grasl-Kraupp B, Holzmann K, Berger W, Wrba F, et al: FGF18 in colorectal tumour cells: Autocrine and paracrine effects. Carcinogenesis 29: 15-24, 2008

17. Flannery CA, Fleming AG, Choe GH, Naqvi H, Zhang M, Sharma A and Taylor HS: Endometrial cancer-associated FGF18 expression is reduced by bazedoxifene in human endometrial stromal cells in vitro and in murine endometrium. Endocrinology 157: 3699-3708, 2016.

18. Forner A: Hepatocellular carcinoma surveillance with miRNAs. Lancet Oncol 16: 743-745, 2015.

19. Hayes CN and Chayama K: MicroRNAs as biomarkers for liver disease and hepatocellular carcinoma. Int J Mol Sci 17: 280, 2016.

20. Shen S, Lin Y, Yuan X, Shen L, Chen J, Chen L, Qin L and Shen B: Biomarker microRNAs for diagnosis, prognosis and treatment of hepatocellular carcinoma: A functional survey and comparison. Sci Rep 6: 38311, 2016.

21. Chen C, Ridzon DA, Broomer AJ, Zhou Z, Lee DH, Nguyen JT, Barbisin M, Xu NL, Mahuvakar VR, Andersen MR, et al: Real-time quantification of microRNAs by stem-loop RT-PCR. Nucleic Acids Res 33: e179, 2005.

22. Livak KJ and Schmittgen TD: Analysis of relative gene expression data using real-time quantitative PCR and the $2^{-\Delta \Delta C_{\mathrm{T}}}$ method. Methods 25: 402-408, 2001.

23. Soifer HS, Rossi JJ and Saetrom P: MicroRNAs in disease and potential therapeutic applications. Mol Ther 15: 2070-2079, 2007.

24. Baker M: RNA interference: MicroRNAs as biomarkers. Nature 464: 1227, 2010.

25. Cui L, Zhang X, Ye G, Zheng T, Song H, Deng H, Xiao B, Xia T, Yu X, Le Y, et al: Gastric juice MicroRNAs as potential biomarkers for the screening of gastric cancer. Cancer 119: 1618-1626, 2013.

26. Li HC, Chen YF, Feng W, Cai H, Mei Y, Jiang YM, Chen T, Xu K and Feng DX: Loss of the Opa interacting protein 5 inhibits breast cancer proliferation through miR-139-5p/NOTCH1 pathway. Gene 603: 1-8, 2017.

27. Krowiorz K, Ruschmann J, Lai C, Ngom M, Maetzig T, Martins V, Scheffold A, Schneider E, Pochert N, Miller C, et al: MiR-139-5p is a potent tumor suppressor in adult acute myeloid leukemia. Blood Cancer J 6: e508, 2016.

28. Yonemori M, Seki N, Yoshino H, Matsushita R, Miyamoto K, Nakagawa $M$ and Enokida H: Dual tumor-suppressors miR-139-5p and miR-139-3p targeting matrix metalloprotease 11 in bladder cancer. Cancer Sci 107: 1233-1242, 2016.

29. Liu H, Yin Y, Hu Y, Feng Y, Bian Z, Yao S, Li M, You Q and Huang Z: miR-139-5p sensitizes colorectal cancer cells to 5-fluorouracil by targeting NOTCH-1. Pathol Res Pract 212: 643-649, 2016.

30. Huang P, Xi J and Liu S: MiR-139-3p induces cell apoptosis and inhibits metastasis of cervical cancer by targeting NOB1. Biomed Pharmacother 83: 850-856, 2016.

31. Sun C, Sang M, Li S, Sun X, Yang C, Xi Y, Wang L, Zhang F, Bi Y, Fu Y, et al: Hsa-miR-139-5p inhibits proliferation and causes apoptosis associated with down-regulation of c-Met. Oncotarget 6: 39756-39792, 2015.

32. Li Q, Liang X, Wang Y, Meng X, Xu Y, Cai S, Wang Z, Liu J and Cai G: miR-139-5p inhibits the epithelial-mesenchymal transition and enhances the chemotherapeutic sensitivity of colorectal cancer cells by downregulating BCL2. Sci Rep 6: 27157, 2016.

33. Chen H, Xu H, Meng YG, Zhang Y, Chen JY and Wei XN: miR-139-5p regulates proliferation, apoptosis, and cell cycle of uterine leiomyoma cells by targeting TPD52. Onco Targets Ther 9: 6151-6160, 2016.

34. Dai S, Wang X, Li X and Cao Y: MicroRNA-139-5p acts as a tumor suppressor by targeting ELTD1 and regulating cell cycle in glioblastoma multiforme. Biochem Biophys Res Commun 467: 204-210, 2015. 